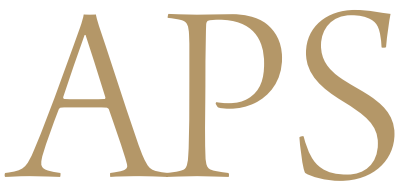

Archives of Plastic Surgery

\title{
Clinical application of cryolipolysis in Asian patients for subcutaneous fat reduction and body contouring
}

\author{
Chang Hyun $\mathrm{Oh}^{1}$, Jeong Su Shim ${ }^{2}$, Kwang Il Bae ${ }^{2}$, Jae Hoon Chang ${ }^{1}$ \\ ${ }^{1}$ BS The Body Aesthetic Plastic Clinic, Busan; ${ }^{2}$ Department of Plastic and Reconstructive Surgery, Catholic University of Daegu School of \\ Medicine, Daegu, Korea
}

Background Cryolipolysis, a preferred method for minimally invasive body contouring, involves the noninvasive cooling of adipocytes to induce lipolysis without damaging other tissues. This study aimed to evaluate the safety and efficacy of cryolipolysis for the treatment of excessive fat tissue.

Methods Between May 2014 and December 2017, 231 patients with 448 areas of interest were enrolled and their records were retrospectively reviewed. We used five different vacuum applicators, and the best-fitting applicator was used for each area. One cycle of cryolipolysis was applied at a cooling intensity factor of 41.6. The efficacy was evaluated 12 weeks after treatment via review of clinical photographs, the pinch test, and ultrasonographic measurements of fat thickness. The occurrence of any complications was also assessed.

Results The volume was reduced in all of the areas to which cryolipolysis was applied. The rate of reduction of the fat layer as measured via the pinch test was $19.2 \%$, and the rate of the decrease in fat layer thickness as measured via ultrasonography was $22.8 \%$. Fat reduction of the upper arm differed significantly from that of the abdomen and flank, but no significant difference was found between sexes. The side effects were limited to erythema, edema, bruising, and numbness at the treatment site and resolved without treatment.

Conclusions Cryolipolysis, with new and better-fitting applicators, is safe, fast, and effective for the reduction of excessive fat tissue on the abdomen, back, flank, and extremities. It is a good option for treating excess adipose tissue in Asian patients.

Keywords Body contouring / Lipoplasty / Subcutaneous fat
Correspondence: Jeong Su Shim Department of Plastic and Reconstructive Surgery, Catholic University of Daegu School of Medicine, 33 Duryugongwon-ro 17-gil, Nam-gu, Daegu 42472, Korea Tel: +82-53-650-4168 Fax: +82-53-650-4584 E-mail: 21csue@hanmail.net

Received: September 21, $2019 \bullet$ Revised: November 5, $2019 \bullet$ Accepted: November 5, 2019

pISSN: 2234-6163 • elSSN: 2234-6171 • https://doi.org/10.5999/aps.2019.01305• Arch Plast Surg 2020;47:62-69

\section{INTRODUCTION}

In modern society, body shape and image can be negatively impacted by unwanted subcutaneous fat. To address this, fat treatment and removal have become staples of the cosmetic market
[1]. Liposuction has been used for a long time and has been regarded as the most effective method for fat treatment, but it does have some disadvantages. It is a surgical procedure that requires anesthesia, which can place strain on the patient, and it has a long recovery time and is accompanied by surgical risks, 
including hematoma, infection, and deep vein thrombosis [2,3]. In recent years, patients have tended to prefer noninvasive body contouring, which has the advantages of a quicker recovery, fewer side effects, and less discomfort [4].

There are many types of noninvasive body contouring, including high-intensity focused ultrasound, radiofrequency treatment, and low-level laser therapy $[5,6]$. Cryolipolysis is a noninvasive method that was approved by the U.S. Food and Drug Administration (FDA) in 2010, and it has been performed by many plastic surgeons because of its body contouring effects via fat reduction [7].

Cryolipolysis induces the apoptosis of adipocytes via the use of selective and noninvasive cooling energy to induce phagocytosis by macrophages. Cryolipolysis is currently used on several areas, including the abdomen, flank, upper and lower extremities, submental area, and male breast, and many studies of its effectiveness are actively being conducted [8-12].

Most previous studies of cryolipolysis included a small number of patients, the majority of whom were Caucasian, and focused on a specific site. In this study, we evaluated the safety and effectiveness of body contouring using cryolipolysis on the abdomen, flank, upper arm, and thigh.

\section{METHODS}

\section{Patients}

This study was performed by retrospectively reviewing the medical charts of patients who underwent cryolipolysis on the abdomen, flank, upper arm, inner thigh, or outer thigh between May 2014 and December 2017. Each patient's medical chart included the patient's age, weight, clinical photographs, and any post-procedure complications, as well as the results of the pinch test and ultrasonography conducted before and after the procedure. The requirement for informed consent was waived, and this investigation was approved by the Institutional Review Board of Daegu Catholic University Medical Center (IRB No. CR-19-152).

Patients with a change in body weight of greater than $5 \%$ after the procedure were excluded from this study. In addition, patients with histories of liposuction or other surgical procedures were also excluded from the study. Moreover, patients with underlying or infectious diseases that could affect the outcome of the surgical procedure and patients with a history of cryoglobulinemia, cold urticaria, or paroxysmal cold hemoglobinuria were also excluded.

\section{Materials}

In this study, we used a cryolipolysis medical device (CoolS- culpting; ZELTIQ Aesthetics, Inc., Pleasanton, CA, USA) for the procedures. The vacuum applicator used depended on the body shape of the patient and the amount of fat present. We used the CoolCore or the CoolMax applicator on the abdominal area, the CoolCurve on the flank, the CoolFit on the inner thigh, the CoolSmooth on the outer thigh, and the CoolFit on the arm. The decision of the applicator that should be used for each body area was made based on the contour, volume, and size of each treatment site.

We marked the peak point, or the point with the fattest tissue in the area to be treated, and then traced the treatment area using the applicator template of the device to be used. According to the protocol developed before the procedure, one cycle of cryolipolysis was applied to each patient at a cooling intensity factor of 41.6. The procedure was performed for 60 minutes with the applicator attached, at a temperature of $11^{\circ} \mathrm{C}$.

After the procedure, the applicator was removed and manual massage was performed. The skin was repeatedly pulled with the fingers in a vigorous kneading motion for the first minute. Then, the tissue was pressed with the fingertip and rubbed in a circular motion for 1 minute.

\section{Evaluation}

Patients who underwent the procedure were instructed to visit our clinic 3 months after the procedure for an evaluation of clinical outcomes. Prior to treatment and at the 3-month follow-up visit, a clinical assessment and the pinch test were conducted, and clinical photographs and ultrasound images were obtained. Clinical photographs were obtained under controlled conditions, using the same light source, room, and camera for the preand post-treatment photographs. Ultrasound images were acquired with a high-resolution linear transducer. A transparent template was created for each patient to ensure that the same area was measured at the 3-month follow-up as was measured prior to treatment, and the thickness of the subcutaneous fat layer was assessed using ultrasound imaging.

The pinch test was performed with the patient in the same posture before and after the procedure to minimize errors. The patient was placed upright in a standing position, and the pinch test was performed on the abdominal area, both flanks, and the inner and outer thighs. In patients whose upper arms were to be treated, both arms were held straight so that the trunk was at a $90^{\circ}$ angle with the upper arm. The skin and subcutaneous fat layer were captured using the thumb and index finger, and caliper measurement was performed with the caliper perpendicular to the skin. The transparent template was used to allow the operator to consistently locate the site or sites to be measured at each evaluation. 


\section{RESULTS}

During the study period, a total of 231 patients ( 184 women and 47 men) were enrolled and completed the treatment, with the exception of subjects who met the exclusion criteria. The mean age of the patients was 39.13 years (range, 24-60 years), with 65 patients between 24 and 29 years, 88 patients between 30 and 39 years, 61 patients between 40 and 49 years, and 17 patients aged 50 years or older.

A total of 448 areas were treated, including 176 cases in the abdomen ( 38 of which were in male subjects), 112 cases in the flank (20 of which were in male subjects), 32 cases in the inner thigh, 48 cases in the outer thigh, and 80 cases in the upper arm. No male subject underwent cryolipolysis of the upper arm or thigh, underscoring the fact that some sex-dependent differences in the areas treated with this procedure were observed.

The pinch test was conducted to measure the fat thickness of the treatment site using calipers prior to treatment and at the 3-month follow-up visit. The mean reduction in fat thickness, as measured by the calipers, was $6 \mathrm{~mm}$ (standard deviation, \pm 2.05 $\mathrm{mm}$ ). The mean fat thickness reduction rate was $19.2 \%$.

Ultrasound measurements were used to calculate the reduction in fat thickness of the treated sites. The mean reduction in fat thickness as measured via ultrasound was $5.31 \mathrm{~mm}$ (standard deviation, $\pm 1.76 \mathrm{~mm}$ ). The mean fat thickness reduction rate was $22.8 \%$. Table 1 shows the vacuum applicators used, the mean values for the reduction in fat thickness, and the mean reduction rates for each area of the body treated. The MannWhitney U-test was performed to assess sex-specific correlations, and the difference in the reduction rate between males and females was not statistically significant $(\mathrm{P}=0.98)$. One-way analysis of variance was performed to compare the treated sites and showed that the reduction rate of the upper arm was significantly different from those of the abdomen and flank $(\mathrm{P}<0.05)$.

Figs. 1-5 show representative subjects before and 3 months after cryolipolysis. The clinical photographs and ultrasound images demonstrated visible changes in the body contours of the treatment sites after the procedures.

Table 1. Fat thickness reduction in each treatment area

\begin{tabular}{|c|c|c|c|c|c|c|}
\hline \multirow[b]{2}{*}{ Area } & \multirow[b]{2}{*}{ No. } & \multirow[b]{2}{*}{ Applicator } & \multicolumn{2}{|c|}{ Caliper } & \multicolumn{2}{|c|}{ Ultrasonography } \\
\hline & & & $\begin{array}{l}\text { Fat thickness reduction } \\
\qquad(\mathrm{mm})\end{array}$ & $\begin{array}{l}\text { Reduction rate } \\
(\%)\end{array}$ & $\begin{array}{l}\text { Fat thickness reduction } \\
\qquad(\mathrm{mm})\end{array}$ & $\begin{array}{c}\text { Reduction rate } \\
(\%)\end{array}$ \\
\hline Abdomen & 176 & CoolCore, CoolMax & $6.2 \pm 2.2$ & 19.6 & $5.3 \pm 1.7$ & 23.2 \\
\hline Flank & 112 & CoolCurve & $6.5 \pm 1.8$ & 20.5 & $5.7 \pm 1.6$ & 23.5 \\
\hline Inner thigh & 32 & CoolFit & $6.1 \pm 1.2$ & 17.9 & $5.3 \pm 1.6$ & 20.6 \\
\hline Outer thigh & 48 & CoolSmooth & $6.2 \pm 2.0$ & 20.5 & $5.5 \pm 1.7$ & 21.9 \\
\hline Upper arm & 80 & CoolFit & $5.1 \pm 2.0$ & 17.3 & $4.6 \pm 1.9$ & 22.8 \\
\hline
\end{tabular}

\section{Fig. 1. Cryolipolysis on the abdomen}

A 45-year-old woman underwent cryolipolysis of her abdominal area to reduce the subcutaneous fat. A visible reduction in the excess fat was seen. (A) A clinical photograph taken prior to treatment. (B) A clinical photograph taken at the 3-month follow-up visit.
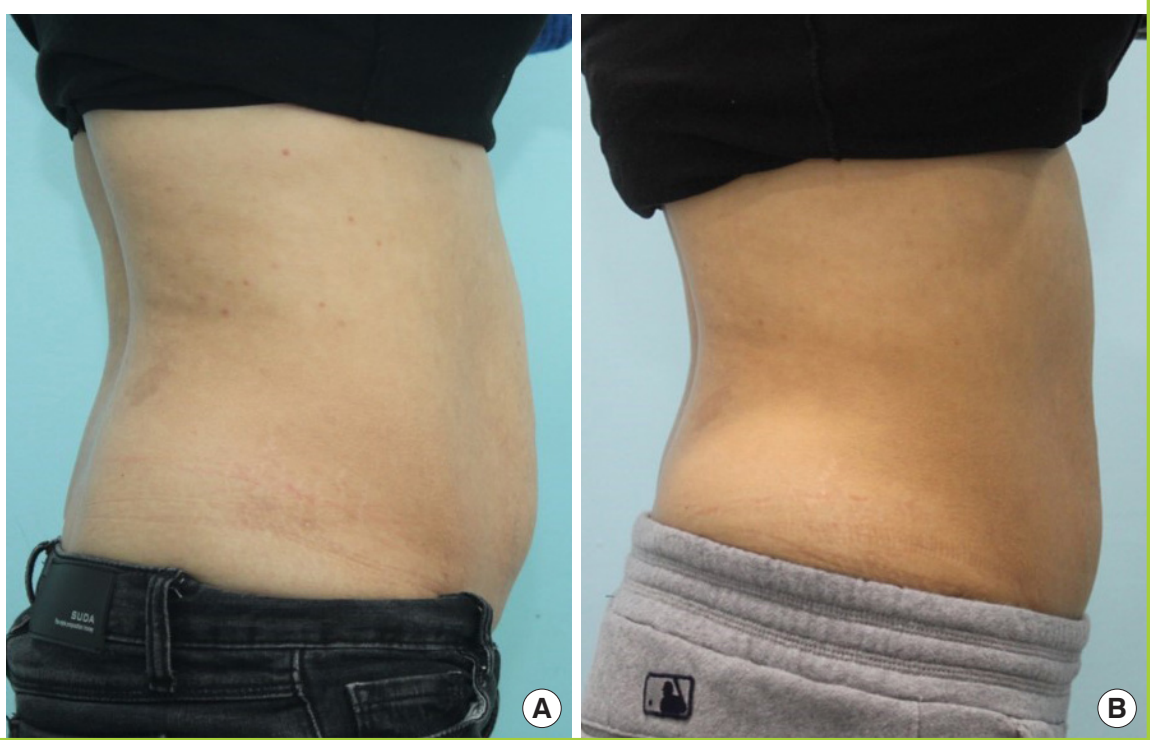


\section{Fig. 2. Cryolipolysis on the lateral thigh}

A 52-year-old woman complained of excess lateral thigh fat and underwent cryolipolysis of the lateral thigh. A visible reduction in the fat bulge was seen. (A) A clinical photograph of the posterior view taken prior to treatment. (B) A clinical photograph of the posterior view taken at the 3-month follow-up visit. (C) A clinical photograph of the oblique view taken prior to treatment. (D) A clinical photograph of the oblique view taken at the 3-month follow-up visit.
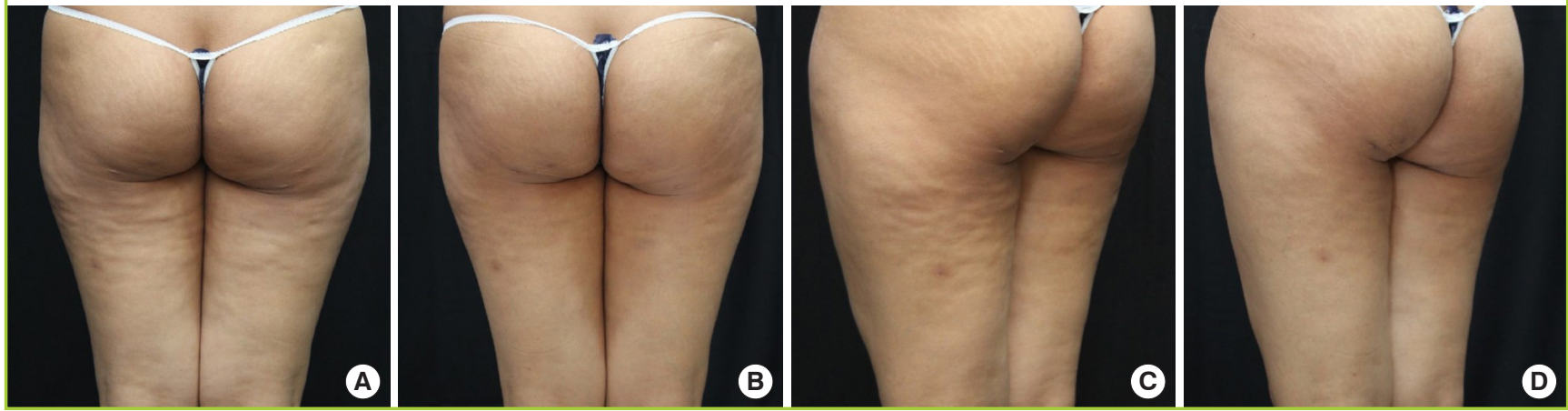

Fig. 3. Cryolipolysis on the upper arm

A 29-year-old woman complained of an extended fat bulge on her upper arm, and she underwent cryolipolysis. A visible reduction in the broad fat bulge of her upper arm was seen. (A) A clinical photograph of the anterior view taken prior to treatment. (B) A clinical photograph of the anterior view taken at the 3-month follow-up visit. (C) A clinical photograph of the posterior view taken prior to treatment. (D) A clinical photograph of the posterior view taken at the 3-month follow-up visit.
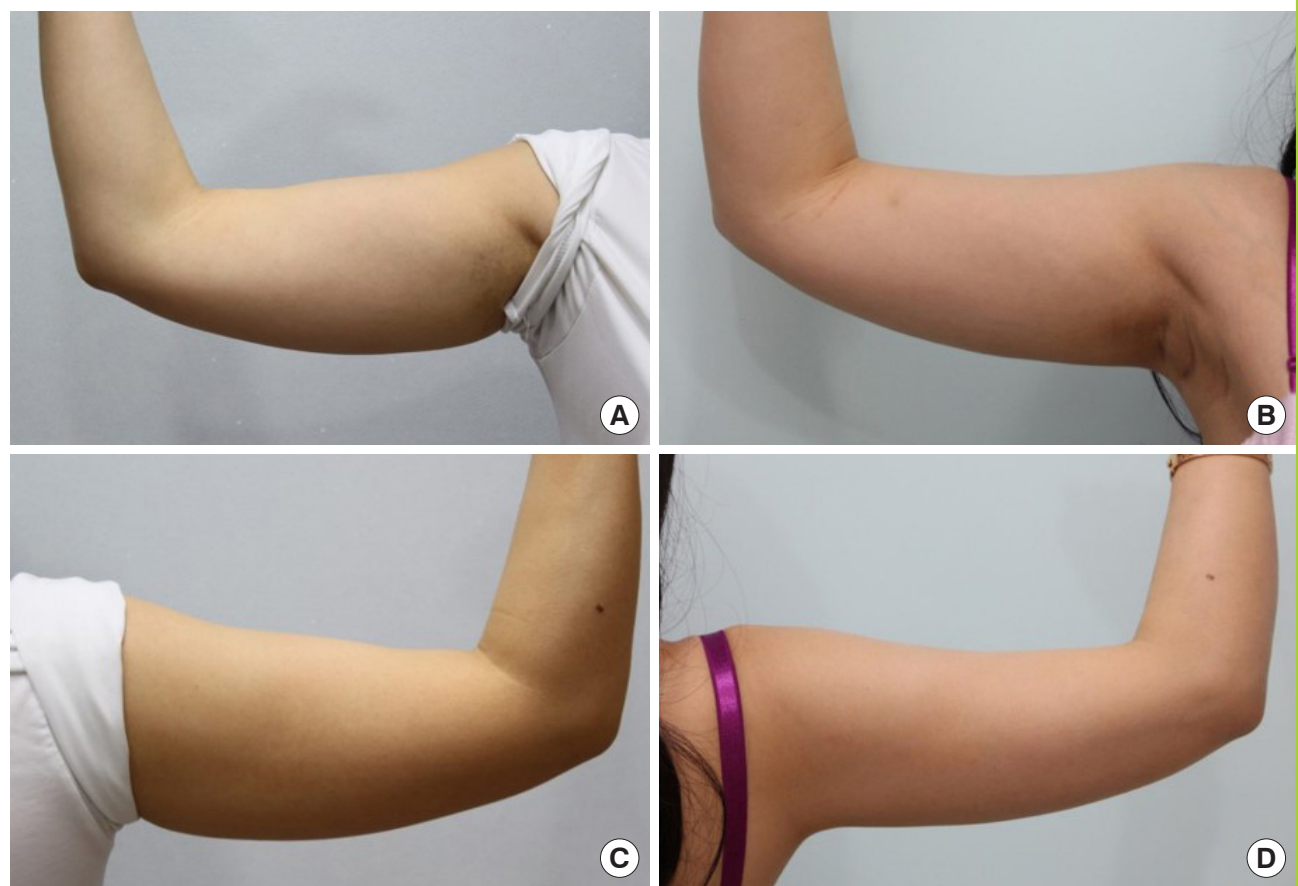

Fig. 4. Representative ultrasound images of the flank

Ultrasound images of a 40-year-old woman who underwent cryolipolysis of the flank. A reduction in the subcutaneous fat under the skin layer was seen. (A) An ultrasound image taken prior to treatment. (B) An ultrasound image taken at the 3-month follow-up visit.
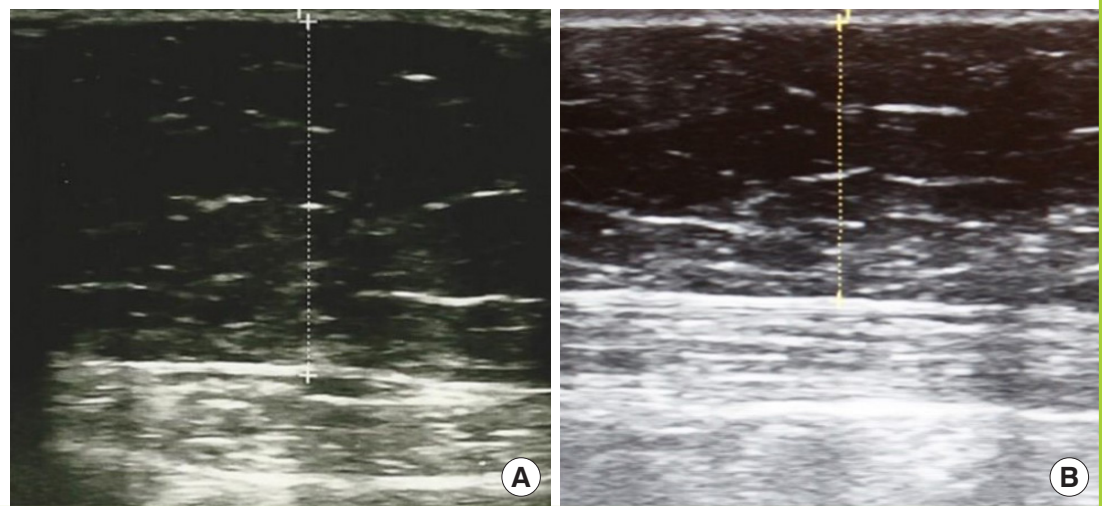


\section{Fig. 5. Representative ultrasound images of the upper arm}

Ultrasound images of a 29 -year-old woman who underwent cryolipolysis of the upper arm. A reduction in the subcutaneous fat layer under the skin was seen. (A) An ultrasound image taken prior to treatment. (B) An ultrasound image taken at the 3-month follow-up visit.

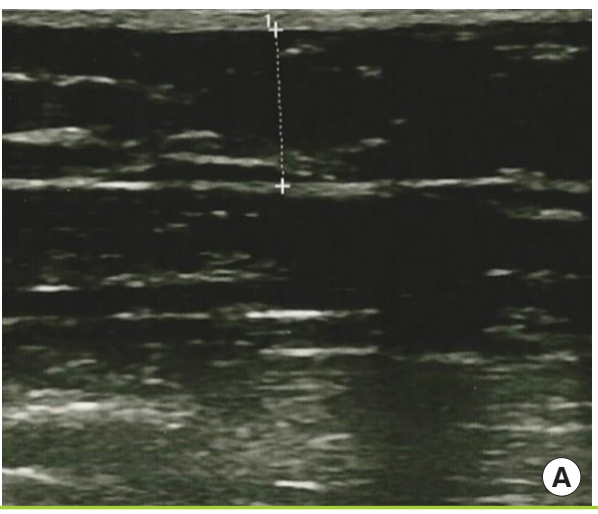

Clinical assessments of the treated sites were performed immediately after each procedure and at each follow-up visit. The side effects of the procedure were temporary and resolved without any specific treatment. Most of the patients who underwent the procedure complained of mild erythema and numbness at the treated sites, which lasted for 1-2 weeks after the procedure. No serious adverse effects were seen.

\section{DISCUSSION}

Liposuction has been the most popular aesthetic procedure in plastic surgery for the last 30 years. Since liposuction was introduced in the 1970s, the tumescent local anesthesia technique has been developed, as have various new techniques, such as laser-assisted, ultrasound-assisted, and radiofrequency-assisted liposuction [5].

Liposuction should be performed under general anesthesia or sedation in order to reduce the discomfort felt by the patient; however, this may place strain on the patient. Moreover, liposuction has a long recovery period with several potential side effects, such as infection, postoperative subcutaneous panniculitis-like reactions, and skin retraction. Above all, the results of liposuction procedures depend on the operator's skill [2,3].

In order to avoid the problems associated with liposuction, many noninvasive body contouring procedures, such as high-intensity focused ultrasound, the radiofrequency technique, and low-level laser therapy, have been developed. Each procedure has its advantages and disadvantages, and the overall situation is ripe for improvement $[5,13]$. Cryolipolysis has been effective for the reduction of subcutaneous fat and remedies some of the described shortcomings of liposuction.

Several previous studies have reported cases of popsicle panniculitis, a type of local inflammation in the fat of an infant's cheek, which confirmed that cold exposure can damage the subcutaneous fat tissue [14]. Early studies of cryolipolysis, initi- ated in 1999, were based on panniculitis. In 2002, Zelickson et al. [15] confirmed the effects of cryolipolysis by reducing the thickness of the fat layer on ultrasound findings and in pathological specimens using a pig model. Later, the safety and efficacy of cryolipolysis was demonstrated in a human model, and clearance from the U.S. FDA was obtained for fat reduction on the flank and abdomen in 2010 and 2012, respectively. Recently, cryolipolysis has been used for subcutaneous fat reduction in various parts of the body, not only on the abdomen, flank, upper extremities, and lower extremities, but also on the buttocks and submental area [8-12].

The mechanism of cryolipolysis has also been studied. The precise application of a cold temperature to a fat layer induces apoptosis of adipocytes, which stimulates the inflammatory process within 3 days of the procedure, resulting in the phagocytosis of the lipids by macrophages and other phagocytes for approximately 1 month. It has been shown that the fat volume decreases after the inflammatory response abates. Because the elimination of adipose tissue in this manner occurs over approximately 3 months [16], the effectiveness of cryolipolysis has been evaluated at 3 months after treatment in many studies, and few studies and data have been published regarding long-term follow-up after cryolipolysis.

Most previous studies were performed with a small number of patients and specific areas of treatment. In this study, we review the results of cryolipolysis on 448 sites in 231 patients, all treated by the same operator in the same clinic. These results were grouped based on the vacuum applicator that was used and the site that was treated. The mean amount of fat thickness reduction as measured using the pinch test and calipers was $6 \mathrm{~mm}$, and the mean fat thickness reduction rate was $19.2 \%$. The mean amount of fat thickness reduction as measured using ultrasound measurements was $5.3 \mathrm{~mm}$, and the mean reduction rate was $22.8 \%$. One-way analysis of variance was performed to compare the treated sites and showed that the reduction rate of the upper 
arm was significantly different from those of the abdomen and flank. Because cryolipolysis acts directly on the subcutaneous fat layer, fat reduction of the upper arms (which have a relatively small amount of fatty tissue) would be expected to be smaller than that of the abdomen or flanks. The Mann-Whitney U-test was performed to assess sex-specific correlation, and the results showed no significant difference between male and female patients.

In most previous studies of cryolipolysis, the majority of subjects were Caucasians. The results of the present study differed from previous cryolipolysis studies in terms of the fat thickness reduction rates observed $[8,10,12]$. A fat thickness reduction was demonstrated in the present study, but it exhibited numerical differences from some studies. Carruthers et al. [17] reported a 3.2-mm fat layer reduction after cryolipolysis on the upper arms, and Zelickson et al. [9] reported a 2.8-mm fat layer reduction on the inner thigh. These differences may have been due to the subject's race, baseline body mass index, or preoperative subcutaneous fat thickness.

Putra et al. [18] performed a review of the literature regarding cryolipolysis in Asian populations and reported that cryolipolysis has displayed good efficacy and safety in Asian patients, although only a few studies, with relatively few subjects, have been performed on Asian populations. In our study, the authors evaluated the efficacy and safety of cryolipolysis based on results obtained from a sufficient number of Asian patients and demonstrated that cryolipolysis has the same efficacy and safety among Asian patients as Putra et al. argued. To identify any differences in the effects of cryolipolysis between Asian and Caucasian patients, further studies may be needed to determine whether other factors, such as skin type or race, can affect the results.

Most of the patients who underwent cryolipolysis complained of transient numbness. Coleman et al. [19] studied the effects of cryolipolysis on the peripheral nerves. The patients in that study exhibited decreases in the response to light touch, two-point discrimination, and temperature sensation for 1-2 weeks after the procedure. Coleman et al. reported that this decrease resolved after 2 months. Based on nerve biopsy results, cryolipolysis was not related to discernable nerve injury, with only minimal effects on the density of the epidermal nerve fibers. The patients in our study complained about mild numbness after the procedure, but this completely resolved without treatment.

There have also been studies of the systemic effects of cryolipolysis on the body. In their 2009 study, Klein et al. [20] reported no significant changes in the serum lipid and liver function tests in patients who underwent cryolipolysis of the flank. These results suggest that cryolipolysis is a safe and well-tolerated pro- cedure.

In this study, the patients underwent cryolipolysis once, and were then followed up 3 months after the procedure. In one previous study, multiple treatments were conducted on the same area of the body. Shek et al. [21] reported that there was a statistically significant improvement when a second treatment was performed 3 months after the first cryolipolysis treatment of the abdominal area. Results from reports and studies involving larger numbers of patients may be necessary; however, that finding indicates that cryolipolysis may be effective even when multiple treatments are performed on the same site.

Cryolipolysis is believed to be an effective procedure for body contouring, but it has some disadvantages. Because cryolipolysis induces the apoptosis of adipocytes by applying cold temperatures, it cannot be used in patients with cold-induced conditions, such as cryoglobulinemia, paroxysmal cold hemoglobinuria, or cold urticaria. Therefore, it is important to obtain an accurate medical history from the patient before the procedure [22].

During the procedure, it is important that the skin is placed in contact with the applicator using negative pressure of the vacuum applicator, if possible. If the skin laxity is low or there is scar tissue at the procedure site, the pain may be severe, and it may be difficult to perform the procedure.

In general, although there may be differences depending on the cosmetic market, cryolipolysis is difficult to access due to its high price.

In 2014, Jalian et al. [23] reported the case of a 40-year-old man with gradual enlargement at the treated site after cryolipolysis. This was a rare case of paradoxical adipose hyperplasia after cryolipolysis. Paradoxical adipose hyperplasia is known to have no spontaneous resolution, and it therefore requires further treatment, such as liposuction or abdominoplasty. No studies have examined the correlation between paradoxical adipose hyperplasia and cryolipolysis, making further studies necessary.

This study evaluated the clinical outcomes at 3 months after cryolipolysis; given the limitation that the long-term results of cryolipolysis are unknown, future studies of the long-term effects of cryolipolysis would be valuable. Additionally, this study was performed as a retrospective review of medical records and had no control group. A further study should be designed to determine any factors-such as the patient's age, body mass index, skin texture, race, or type of applicator-that may influence the clinical outcomes. Such a study should preferably incorporate objective evaluation methods instead of caliper measurements, which yield imprecise values of fat thickness.

The results of this study indicate that subcutaneous fat was effectively removed through cryolipolysis, with no permanent 
complications that required specific treatment. Therefore, cryolipolysis is safe, fast, and effective for the reduction of excessive fat tissue on the abdomen, back, flank, and extremities.

\section{NOTES}

\section{Conflict of interest}

No potential conflict of interest relevant to this article was reported.

\section{Ethical approval}

The study was approved by the Institutional Review Board of Daegu Catholic University Medical Center (IRB No. CR-19152) and performed in accordance with the principles of the Declaration of Helsinki.

\section{Patient consent}

The patients provided written informed consent for the publication and the use of their images.

\section{Author contribution}

Conceptualization: Oh CH, Shim JS, Bae KI, Chang JH. Data curation: Oh CH, Shim JS, Bae KI, Chang JH. Formal analysis: Oh CH, Shim JS, Bae KI, Chang JH. Methodology: Oh CH, Shim JS, Bae KI, Chang JH. Project administration: $\mathrm{Oh} \mathrm{CH}$, Shim JS, Bae KI, Chang JH. Visualization: Shim JS, Bae KI.Writing - original draft: Shim JS, Bae KI. Writing - review \& editing: Oh CH, Shim JS, Bae KI, Chang JH.

\section{ORCID}

Chang Hyun Oh https://orcid.org/0000-0002-8919-1741

Jeong Su Shim https://orcid.org/0000-0002-8458-4415

Kwang Il Bae https:/ / orcid.org/0000-0003-4496-2159

Jae Hoon Chang https://orcid.org/0000-0002-0909-1602

\section{REFERENCES}

1. Ahmad J, Eaves FF 3rd, Rohrich RJ, et al. The American Society for Aesthetic Plastic Surgery (ASAPS) survey: current trends in liposuction. Aesthet Surg J 2011;31:214-24.

2. Heymans O, Castus P, Grandjean FX, et al. Liposuction: review of the techniques, innovations and applications. Acta Chir Belg 2006; 106:647-53.

3. Desrosiers AE 3rd, Grant RT, Breitbart AS. Don't try this at home: liposuction in the kitchen by an unqualified practitioner leads to disastrous complications. Plast Reconstr Surg 2004;113:460-1.

4. Holliday R, Elfving-Hwang J. Gender, globalization and aes- thetic surgery in South Korea. Body Soc 2012;18:58-81.

5. Mulholland RS, Paul MD, Chalfoun C. Noninvasive body contouring with radiofrequency, ultrasound, cryolipolysis, and low-level laser therapy. Clin Plast Surg 2011;38:503-20.

6. Katz B, McBean J, Cheung JS. The new laser liposuction for men. Dermatol Ther 2007;20:448-51.

7. Avram MM, Harry RS. Cryolipolysis for subcutaneous fat layer reduction. Lasers Surg Med 2009;41:703-8.

8. Rivers JK, Ulmer M, Vestvik B, et al. A customized approach for arm fat reduction using cryolipolysis. Lasers Surg Med 2018;50;732-7.

9. Zelickson BD, Burns AJ, Kilmer SL. Cryolipolysis for safe and effective inner thigh fat reduction. Lasers Surg Med 2015;47:120-7.

10. Stevens WG, Bachelor EP. Cryolipolysis conformable-surface applicator for nonsurgical fat reduction in lateral thighs. Aesthet Surg J 2015;35:66-71.

11. Suh DH, Park JH, Jung HK, et al. Cryolipolysis for submental fat reduction in Asians. J Cosmet Laser Ther 2018;20:247.

12. Munavalli GS, Panchaprateep R. Cryolipolysis for targeted fat reduction and improved appearance of the enlarged male breast. Dermatol Surg 2015;41:1043-51.

13. Caruso-Davis MK, Guillot TS, Podichetty VK, et al. Efficacy of low-level laser therapy for body contouring and spot fat reduction. Obes Surg 2011;21:722-9.

14. Ter Poorten JC, Hebert AA, Ilkiw R. Cold panniculitis in a neonate. J Am Acad Dermatol 1995;33(2 Pt 2):383-5.

15. Zelickson B, Egbert BM, Preciado J, et al. Cryolipolysis for noninvasive fat cell destruction: initial results from a pig model. Dermatol Surg 2009;35:1462-70.

16. Nelson AA, Wasserman D, Avram MM. Cryolipolysis for reduction of excess adipose tissue. Semin Cutan Med Surg 2009;28:244-9.

17. Carruthers J, Stevens WG, Carruthers A, et al. Cryolipolysis and skin tightening. Dermatol Surg 2014;40 Suppl 12:S1849.

18. Putra IB, Jusuf NK, Dewi NK. Utilisation of cryolipolysis among Asians: a review on efficacy and safety. Open Access Maced J Med Sci 2019;7:1548-54.

19. Coleman SR, Sachdeva K, Egbert BM, et al. Clinical efficacy of noninvasive cryolipolysis and its effects on peripheral nerves. Aesthetic Plast Surg 2009;33:482-8.

20. Klein KB, Zelickson B, Riopelle JG, et al. Non-invasive cryolipolysis for subcutaneous fat reduction does not affect serum lipid levels or liver function tests. Lasers Surg Med 2009; 41:785-90.

21. Shek SY, Chan NP, Chan HH. Non-invasive cryolipolysis 
for body contouring in Chinese: a first commercial experience. Lasers Surg Med 2012;44:125-30.

22. Meyer PF, Davi Costa E Silva J, Santos de Vasconcellos L, et al. Cryolipolysis: patient selection and special consider- ations. Clin Cosmet Investig Dermatol 2018;11:499-503.

23. Jalian HR, Avram MM, Garibyan L, et al. Paradoxical adipose hyperplasia after cryolipolysis. JAMA Dermatol 2014; 150:317-9. 